\title{
A NOVEL HYBRID APPROACH FOR THE EXTRACTION OF LINEAR/CYLINDRICAL FEATURES FROM LASER SCANNING DATA
}

\author{
Z. Lari and A. Habib \\ Department of Geomatics Engineering, University of Calgary, Calgary, Alberta, Canada T2N 1N4 - \\ (zlari, ahabib@ucalgary.ca)
}

Commission III, WG III/2

KEY WORDS: Laser scanning, Point cloud, Feature extraction, Modelling, Segmentation, Performance

\begin{abstract}
:
Laser scanning systems have been widely adopted for directly providing 3D point cloud over physical surfaces at high density. However, the collected point cloud should undergo manipulation approaches to be utilized for diverse civil, industrial, and military applications. Different processing techniques have consequently been implemented for the extraction of low-level features from this data. Linear/cylindrical features are among the most important primitives that could be extracted from laser scanning data, especially those collected in industrial sites and urban areas. This paper presents a novel approach for the identification, parameterization, and segmentation of these features in a laser point cloud. In the first step of the proposed approach, the points which belong to linear/cylindrical features are detected and their appropriate representation models are chosen based on the principal component analysis of their local neighborhood. The approximate direction and position parameters of the identified linear/cylindrical features are then refined using an iterative line/cylinder fitting procedure. A parameter-domain segmentation method is finally applied to isolate the points which belong to individual linear/cylindrical features in direction and position attribute spaces, respectively. Experimental results from real datasets will demonstrate the feasibility of the proposed approach for the extraction of linear/cylindrical features from laser scanning data.
\end{abstract}

\section{INTRODUCTION}

Over the past few years, laser scanning systems have been acknowledged as cost-effective and reliable tools for rapid collection of high density 3D point cloud over physical surfaces. The advent of these systems has reduced the cost and increased the accessibility to accurate 3D data for different applications such as 3D city modelling, transportation planning, emergency response, cultural heritage documentation, military simulation, structural health monitoring, and industrial site modelling. The original laser scanning point cloud usually does not exhibit semantic information about the type and characteristics of the scanned surfaces. Therefore, the collected data should be processed to extract the required information for the aforementioned applications. The identification of low-level geometric primitives is often the primary step in information extraction from a laser point cloud. Linear/cylindrical features are among the most important primitives that could be encountered in laser scanning data, especially those collected in urban areas, industrial sites, and electrical substations. As a result, automatic and robust methods are essential for the detection and extraction of these features from laser scanning data.

To date, a variety of techniques have been proposed for the detection and segmentation of linear/cylindrical features in laser point cloud. These methods can be mainly categorized into three groups: 1) The methods which try to extract the linear/cylindrical features in spatial domain, 2) The methods which identify the linear/cylindrical features in parameter space, and 3) hybrid methods. In the spatial-domain techniques, a principal component analysis of the local neighborhood of individual laser points is initially performed to classify the points which belong to linear/cylindrical features and approximate their geometric properties (Luk'acs et al., 1998; Marshall et al., 2001; Belton and Lichti, 2006; Gross and Thoennessen, 2006; El-Halawany and Lichti, 2011). In some of the approaches in this category, a non-linear least squares procedure is also employed to precisely define the geometric properties of the best fitted lines/cylinders to the classified linear/cylindrical surfaces. A region growing algorithm is then implemented utilizing arbitrary seed points to cluster the points which potentially belong to linear/cylindrical features. This procedure aggregates the points with similar geometric characteristics and defines the entire connected linear/cylindrical features. These methods are able to reliably classify and extract the linear/cylindrical features. However, their outcome is highly sensitive to the size of the established local neighborhoods. The other drawback of these approaches is the dependency of their performance on the selection of seed points. It is usually difficult to judge if the selection of one set of seed points may lead to better segmentation results than the other.

In the parameter-domain techniques, the geometric attributes which describe linear/cylindrical features - cylinder axis direction (two parameters), cylinder position (two parameters), and cylinder radius (one parameter) - are initially estimated for local neighborhoods. A five-dimensional attribute space will then be established for the extraction of linear/cylindrical features. In such an attribute space, the detection and extraction of linear/cylindrical features will be time and memory consuming. In order to reduce the computational cost of this procedure, the linear/cylindrical features are detected in the two-dimensional direction and three-dimensional position and radius attribute subspaces, respectively (Kimme et al., 1975; Vosselman 2004; Rabbani and Heuvel, 2005; Rabbani, 2006). In the first step of this sequential approach, a Gaussian sphere of the laser point cloud is constructed and a two-dimensional Hough transform is performed to identify the cylindrical features which have similar axis direction in the established Gaussian sphere. In the second step, a three-dimensional Hough transform is carried out to identify linear/cylindrical features based on their position and radius parameters. Although this procedure greatly improves the performance of the parameterdomain linear/cylindrical feature extraction procedure, it is still computationally inefficient to process a massive amount of points simultaneously. 
In the existing hybrid approaches, the linear/cylindrical features are initially classified in the parameter domain and then precisely modelled using least squares line/cylinder fitting procedure in the spatial domain (Bolles and Fischler, 1981; Fischler and Bolles, 1987; Chaperon and Goulette, 2001). These methods usually employ a RANdom SAmple Consensus (RANSAC) algorithm on a Gaussian sphere to approximate the axis direction of linear/cylindrical features. The estimated direction parameters will be then utilized as the initial values for the least squares line/cylinder fitting (Chaperon and Goulette, 2001). The limitation of these approaches is that they cannot reliably identify multiple linear/cylindrical features with different radius parameters along one direction.

In order to overcome the limitations of the previouslymentioned approaches, an alternative hybrid approach for the segmentation and extraction of linear/cylindrical features from laser scanning data is presented in this paper. In this approach, linear/cylindrical features with different representation forms are firstly detected and modelled in the spatial domain. A parameter-domain segmentation technique is then implemented to extract the linear/cylindrical features in low-dimensional attribute subspaces constructed for each representation form. The proposed methodology for linear/cylindrical features detection, representation, and segmentation is discussed in section 2 in detail. The performance of the proposed approach for linear/cylindrical feature extraction is then assessed through experimental results using real laser scanning data in section 3 . Finally, concluding remarks and recommendations for future research work are provided in section 4 .

\section{METHODOLOGY}

In this section, the proposed methodology for the automated detection and extraction of linear/cylindrical features from laser scanning data will be described. This approach is implemented in three steps. In the first step, a principal component analysis procedure is performed to classify the laser point cloud and detect the points which belong to linear/cylindrical features. The appropriate representation forms for the detected linear/cylindrical features are then selected based on the estimated eigen values from the principal component analysis. In the second step, the geometric attributes which model the detected linear/cylindrical features with different representation forms are approximated and then precisely estimated using an iterative line/cylinder fitting procedure. In the final step, the estimated attributes are clustered in direction and position parameter domains, sequentially. Figure 1 shows the outline of the proposed methodology. The detailed explanation of these steps is presented in the following sub-sections.

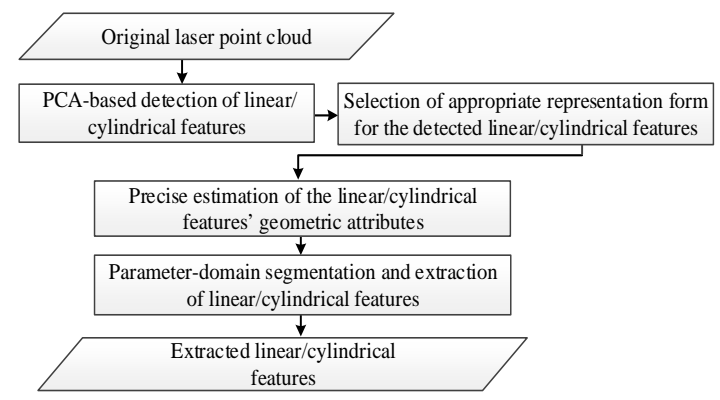

Figure 1. The outline of the proposed methodology

\subsection{Detection of linear/cylindrical features}

In the first step of the proposed methodology, the points which belong to linear/cylindrical features should be detected in the laser point cloud. Therefore, the principal component analysis is initially utilized to classify the laser scanning points based on their geometric properties in the spatial domain (Pauly et al, 2002). This procedure commences by defining a spherical neighborhood of a query point. This neighborhood encloses a predefined number $(n)$ of the nearest neighbors of the query point. Afterwards, the nature of the point cloud within the established neighborhood - planarity, linearity, or roughness is investigated through eigenvalue analysis of the dispersion matrix of the points within that neighborhood relative to their centroid. This dispersion matrix (Cov) is given by:

$$
\operatorname{Cov}=\frac{1}{n+1} \sum_{i=1}^{n+1}\left(\vec{r}_{i}-\vec{r}_{C}\right)\left(\vec{r}_{i}-\vec{r}_{C}\right)^{T}
$$

Where,

$$
\vec{r}_{i}=\left[\begin{array}{lll}
X_{i} & Y_{i} & Z_{i}
\end{array}\right]^{T}
$$

and,

$$
\vec{r}_{C}=\frac{1}{n+1} \sum_{i=1}^{n+1} \vec{r}_{i}
$$

The eigen value decomposition of the dispersion matrix results in two matrices $(\Lambda, W)$ whose components are the eigenvalues $\left(\lambda_{1}, \lambda_{2}, \lambda_{3}\right)$ and their corresponding eigenvectors $\left(\vec{e}_{1}, \vec{e}_{2}, \vec{e}_{3}\right)$.

$$
\operatorname{Cov}=W \Lambda W^{T}=\left[\begin{array}{lll}
\vec{e}_{1} & \vec{e}_{2} & \vec{e}_{3}
\end{array}\right]\left[\begin{array}{ccc}
\lambda_{1} & 0 & 0 \\
0 & \lambda_{2} & 0 \\
0 & 0 & \lambda_{3}
\end{array}\right]\left[\begin{array}{l}
\vec{e}_{1}^{T} \\
\vec{e}_{2}^{T} \\
\vec{e}_{3}^{T}
\end{array}\right]
$$

The estimated eigen vectors represent the principal components (orientation) of the established neighborhood while the eigen values denote the variance (extent) in the direction of their corresponding eigen vector. These eigen values are then normalized and utilized for the classification of local neighborhoods of individual points. For linear neighborhoods, one of the normalized eigen values will be quite larger (almost equal to 1) when compared to the other two eigen values (approximately equal to zero). The query points which have such eigen values will be detected as a part of linear/cylindrical feature (Figure 2).

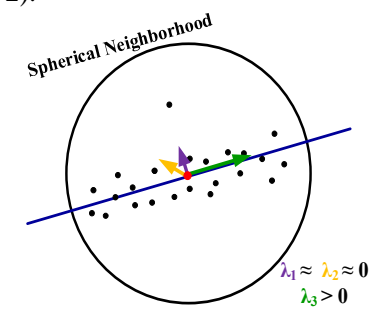

Figure 2. 2D representation of the established spherical neighborhood including a linear/cylindrical feature

In linear/cylindrical neighborhoods, the orientation of the eigen vector which corresponds to the largest eigen value will represent the approximate direction of the detected linear/cylindrical feature. The appropriate representation models for the detected linear/cylindrical features can be then chosen based on their approximated directions through the principal component analysis procedure.

\subsection{Modelling the detected linear/cylindrical features and segmentation attributes computation}

Once the laser scanning points which belong to linear/cylindrical features have been detected using the principal component analysis procedure, appropriate representation models should be selected to parametrically describe these features. As mentioned in the previous subsection, the direction of the detected linear/cylindrical features axis can be approximated by the eigen vector which corresponds to the largest eigen value estimated through principal component 
analysis. This approximate axis direction is then utilized as the basis for the selection of the appropriate representation models for the detected linear/cylindrical features.

In order to avoid singularities in linear/cylindrical features representation and reduce the number of the required parameters for feature extraction, three representation models are utilized to define each of the detected linear/cylindrical features:

1. The first representation form models linear/cylindrical features which are not parallel to XY-plane (for which the Z component of the eigenvector $\left(e_{Z}\right)$ corresponding to the largest eigenvalue is larger than its $\mathrm{X}$ and $\mathrm{Y}$ components $\left.\left(e_{X}, e_{Y}\right)\right)$. The intersection point of these linear/cylindrical features with the $\mathrm{XY}$-plane $(p, q, 0)$ as well as their direction vector $(a, b, 1)$ are utilized to represent these features in the spatial domain (Equation 3). $t$ is the parameter that describes the point location along the linear/cylindrical feature axis.

$$
\left\{\begin{array}{c}
X=a t+p \\
Y=b t+q \\
Z=t
\end{array}\right.
$$

2. The second representation form models linear/cylindrical features which are not parallel to XZ-plane (for which the Y component of the eigenvector $\left(e_{Y}\right)$ corresponding to the largest eigenvalue is larger than its $\mathrm{X}$ and $\mathrm{Z}$ components $\left.\left(e_{X}, e_{Z}\right)\right)$. The intersection point of these linear/cylindrical features with the XZ-plane $(p, 0, q)$ as well as their direction vector $(a, 1, b)$ are utilized to represent these features in the spatial domain (Equation 4). $t$ is the parameter that describes the point location along the linear/cylindrical feature axis.

$$
\left\{\begin{array}{c}
X=a t+p \\
Y=t \\
Z=b t+q
\end{array}\right.
$$

3. The third representation form models linear/cylindrical features which are not parallel to YZ-plane (for which the $\mathrm{X}$ component of the eigenvector $\left(e_{X}\right)$ corresponding to the largest eigenvalue is larger than its $\mathrm{Y}$ and $\mathrm{Z}$ components $\left(e_{Y}, e_{Z}\right)$ ). The intersection point of these linear/cylindrical features with the YZ-plane $(0, p, q)$ as well as their direction vector $(1, a, b)$ are utilized to represent these features in the spatial domain (Equation 5). $t$ is the parameter that describes the point location along the linear/cylindrical feature axis.

$$
\left\{\begin{array}{c}
X=t \\
Y=a t+p \\
Z=b t+q
\end{array}\right.
$$

Once the geometric parameters defining the linear/cylindrical features $(a, b, p$, and $q)$ are approximated through the principal component analysis, an adaptive cylindrical neighbourhood (Figure 3) is defined to precisely estimate the parameters describing the linear/cylindrical features. The height of this adaptive cylinder is specified by the distance between the query point and its $n^{\text {th }}$-nearest neighbour. The orientation of the cylinder axis - direction of the best-fitted line/cylinder axis- is then derived through an iterative line/cylinder fitting procedure using generalized least squares adjustment. This procedure aims at minimizing the squared sum of the normal distances $\left(d_{n i}\right)$ between the points in the spherical neighborhood and linear/cylindrical feature in question using the target function stated in Equation 6. In order to reduce the impact of the outliers in this iterative cylinder fitting procedure, the points within the spherical neighborhood are assigned weights $\left(w_{i}\right)$ that are inversely proportional to their normal distances from the best fitted cylinder's surface in the previous iteration.

$$
\sum_{i=1}^{n} w_{i}^{2} d_{n_{i}}^{2}=\min \left(u_{X}, u_{Y}, u_{Z}, X_{0}, Y_{0}, Z_{0}, R\right)
$$

In this target function, $n$ is the number of the points used to define the linear/cylindrical feature, $\left(u_{X}, u_{Y}, u_{Z}\right)$ are the direction parameters of the linear/cylindrical feature in question which corresponds to either $(a, b, 1),(a, 1, b)$, or $(1, a, b)$, and $\left(X_{0}, Y_{0}\right.$, $Z_{0}$ ) are the parameters which define the position of a point on the line/cylinder axis which may correspond to either $(p, q, 0)$, $(p, 0, q)$, or $(0, p, q)$, and $(R)$ is the cylinder radius which will be equal to 0 for linear features.

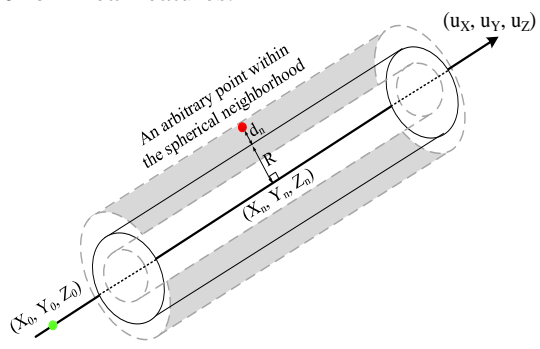

Figure 3. The established adaptive cylindrical neighbourhood for linear/cylindrical features definition (for linear features, $\mathrm{R}=0$ )

The major advantages of this approach, when compared to the principal component analysis, are filtering out the outliers in the iterative cylinder fitting procedure and simultaneous estimation of the direction, position, and radius parameters which represent linear/cylindrical features. The estimated geometric parameters $\left(u_{X}, \quad u_{Y}, u_{Z}, \quad X_{0}, \quad Y_{0}, \quad Z_{0}, R\right)$ describing the detected linear/cylindrical features will be utilized in a parameter-domain segmentation approach to isolate and extract the points that belong to individual linear/cylindrical features.

\subsection{Parameter-domain segmentation and extraction of linear/cylindrical features}

So far, we have discussed the proposed approaches for the detection and appropriate modelling of the linear/cylindrical features in a laser point cloud. In this section, a new parameterdomain method is introduced to segment and aggregate the points which belong to linear/cylindrical features with similar geometric parameters representing their axes $\left(u_{X}, u_{Y}, u_{Z}, X_{0}, Y_{0}\right.$, $Z_{0}$ ). Therefore, the clusters of the points with similar attributes should be detected in a six-dimensional attribute space. The high dimension of the established attribute space makes it almost impractical to detect these clusters efficiently. Hence, the new proposed approach tries to overcome the computational inefficiency (i.e., time and memory consumption problems) of available parameter-domain approaches for the segmentation of linear/cylindrical features in a high-dimensional attribute spaces. This alternative approach is implemented to reduce the computational volume and improve the performance of the parameter-domain segmentation procedure.

The proposed alternative for improving the performance of the parameter-domain segmentation approach is to discriminate the individual linear/cylindrical features in directional and positional attribute subspaces, sequentially. Since the linear/cylindrical features' axes modelled using one of the representation forms, have two directional $(a, b)$ and two positional $(p, q)$ degrees of freedom, the dimension of directional and positional attribute subspaces is reduced from three to two.

In the first phase of this sequential cluster detection procedure, the estimated directional parameters $(a, b)$, for the linear/cylindrical features' axes modelled by one of the representation forms, are firstly organized in a two-dimensional kd-tree structure which is manipulated for peak detection. In order to detect and extract the clusters of points in the established parameter domain, an appropriate extent of the 
cluster $(r)$ for each attribute point should be initially estimated while considering the acceptable angular deviation $(\Delta \alpha)$ among the collinear points. In order to estimate these extents, we should firstly evaluate the maximum variations in their direction parameters $\left(\Delta a_{\max }, \Delta b_{\max }\right)$ due to an acceptable angular deviation $\Delta \alpha$. For example, for a linear/cylindrical feature which has been modelled by the first representation form, these variations are estimated using Equation 7 by minimizing one of these variations and computing the maximum value for the other one. Based on the estimated $\Delta a_{\max }$ and $\Delta b_{\max }$, the cluster extent $(r)$ is computed using Equation 8.

$$
\begin{gathered}
{\left[\begin{array}{l}
a \\
b \\
1
\end{array}\right] \cdot\left[\begin{array}{c}
a+\Delta a \\
b+\Delta b \\
1
\end{array}\right]=\cos (\Delta \alpha) \sqrt{a^{2}+b^{2}+1} \sqrt{(a+\Delta a)^{2}+(b+\Delta b)^{2}+1}} \\
r=\sqrt{\Delta a_{\max }^{2}+\Delta b_{\max }^{2}}
\end{gathered}
$$

Once the appropriate cluster extent estimated, all the points in the direction parameter domain are checked to count the number of their neighboring points within their established cluster extent. The attribute point with the highest neighbors count is chosen as the first peak. The points in the spatial domain whose attributes are the constituents of the detected peak are then considered as one segment and removed from the direction parameter domain. These points represent parallel linear/cylindrical features in the spatial domain. The search for the highest count peak is repeated until the number of detected attribute points in the identified peak is less than the minimum number of points required for reliable linear/cylindrical feature definition. Figure 4 shows the peak detection procedure in the direction parameter domain.

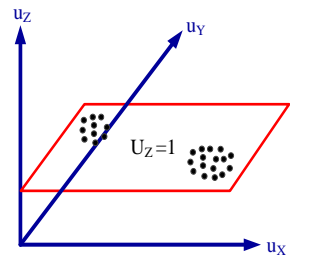

(a)

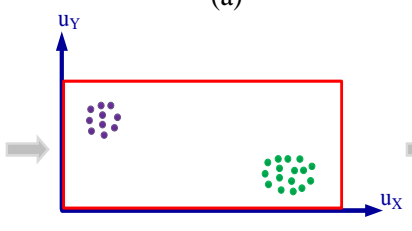

(c)

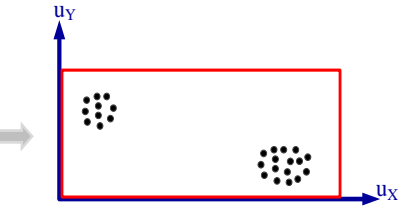

(b)
Figure 4.The peak detection procedure in the direction parameter-domain: (a) 3D direction parameter domain, (b) 2D direction parameter domain, (c) detected peaks in the direction parameter domain, (d) parallel lines representing the detected peaks in the spatial domain

In the second phase of this sequential procedure, the position parameters $(p, q)$ of the points whose direction parameters are detected as one cluster in the directional parameter domain are organized in a 2D kd-ree structure which is manipulated for peak detection. All the points in the established positional parameter domain are then checked to count the number of their neighboring points within their established cluster extent. In the positional parameter domain, the extent of the clusters is defined as $(\Delta d / \sin (\alpha))$ where $\Delta d$ is the acceptable distance between collinear points and $\alpha$ is the angle between the line and the plane where the intersection point takes place. The attribute point with the highest neighbors count is chosen as the first peak in the positional parameter domain. The points in the spatial domain whose attributes are the constituents of the detected peak are then considered as one segment and removed from the positional parameter domain. These points represent an isolated linear/cylindrical feature in the spatial domain. The search for the highest count peaks is then continued until the number of detected attribute points in the identified peak is less than the minimum number of points required for reliable linear/cylindrical feature definition. Figure 5 shows the peak detection and linear/cylindrical features extraction from the positional parameter domain.

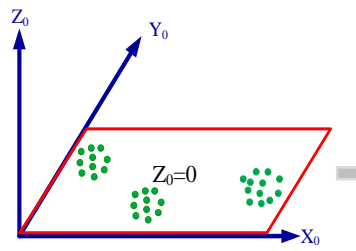

(a)

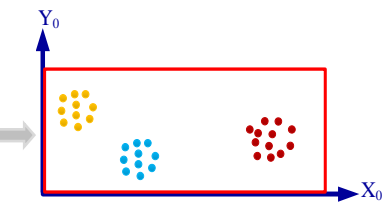

(c)

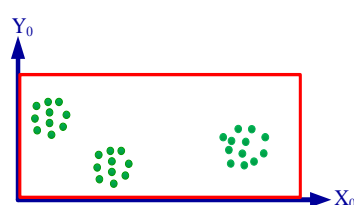

(b)
Figure 5. The peak detection and linear/cylindrical features extraction from the position parameter domain: (a) 3D position parameter domain for a detected peak in the direction parameter domain, (b) 2D position parameter domain for a detected peak in the direction parameter domain, and c) detected peaks in the position parameter domain, and (d) extracted lines representing the detected peaks in the spatial domain

This procedure is repeated for all of the detected linear/cylindrical features with different representation forms to extract and isolate the possible linear/cylindrical features in a laser point cloud. The implementation of the introduced approach will improve the performance of the proposed parameter-domain methodology for linear/cylindrical features extraction by sequential segmentation of these features in lowerdimensional directional and positional attribute spaces. The estimated cylinder radius $(R)$ can be later utilized in a onedimensional parameter domain for the discrimination of collinear linear and cylindrical features, and coaxial cylindrical features.

\section{EXPERIMENTAL RESULTS}

To verify the performance of the proposed methodology for the extraction of linear/cylindrical features, a set of experiments using two terrestrial laser scanning datasets have been conducted. The first terrestrial laser dataset (Figure 6.a) was acquired in an electrical substation using a FARO Focus ${ }^{3 \mathrm{D}}$ terrestrial laser scanner. The second terrestrial laser dataset was obtained from a complex building and its surrounding area (Figure 6.b) using a Trimble GS200 terrestrial laser scanner. The first dataset includes a variety of linear/cylindrical features such as light poles, electrical transmission lines, electrical transformers, and surge arresters. The second dataset, on the other hand, contains only a few linear/cylindrical features such as light poles and fences. These datasets are processed using the proposed approach to extract individual linear/cylindrical features. Table 1 lists the utilized thresholds for the extraction of linear/cylindrical features from these datasets. These thresholds can be easily derived using rough knowledge about the nature of the scanned area and the utilized scanner. 


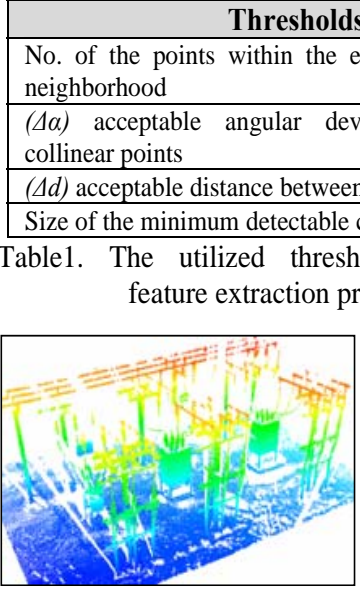

(a) (b)

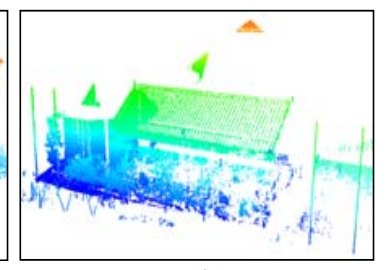

Figure 6. Original laser scanning datasets: (a) electrical substation dataset, and (b) complex building and surrounding area dataset

In order to illustrate the performance of the proposed hybrid segmentation procedure in the direction and position attribute subspaces, a small section of the electrical substation laser point cloud (Figure 7.a) has been initially selected and processed. Figure 7.b shows the outcome of the linear/cylindrical feature detection procedure, where the laser points which have been detected as being part of linear/cylindrical features by the principal component analysis are shown in red color. Figure $7 . c$ shows the linear/cylindrical features which have been clustered as a peak in the direction attribute subspace in blue color (in the spatial domain). Figure 7.d shows an isolated linear/cylindrical feature which has been clustered as a peak in the positional attribute subspace in green color. Figure 7.e shows the linear/cylindrical features which have been ultimately extracted using the proposed feature extraction procedure.

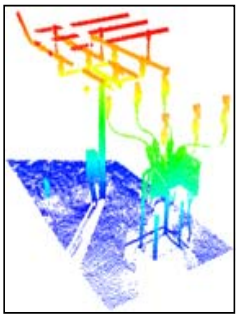

(a)

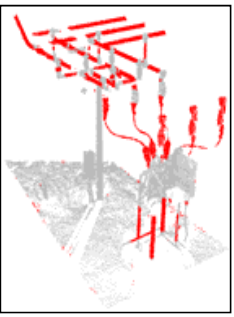

(b)

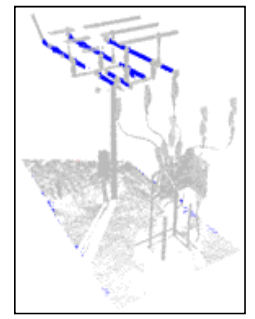

(c)

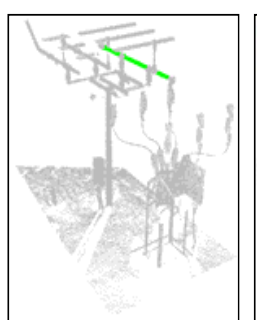

(d)

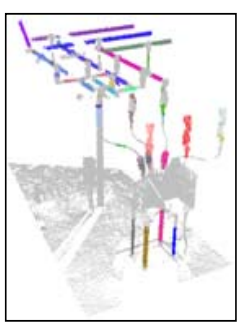

(e)
Figure 7. The intermediate results of the proposed linear/cylindrical feature extraction procedure: (a) original point cloud, (b) detected linear/cylindrical features from the principal component analysis (red points), (c) parallel linear/cylindrical features representing a detected peak in the direction attribute subspace, (d) a linear/cylindrical feature representing a detected peak in the position attribute subspace, and (e) extracted linear/cylindrical features

The linear/cylinder feature extraction procedure is then carried out for the two aforementioned laser datasets (Figures 6.a and 6.b) in two steps: In the first step, the linear/cylindrical features are detected and modelled in the spatial domain using the principal components analysis. Figures 8.a and 9.a show the detected linear cylindrical features in these datasets in red color. The detected linear/cylindrical features are then segmented using the proposed parameter-domain segmentation approach. Figures 8.b and 9.b show the extracted linear features in the laser point clouds.

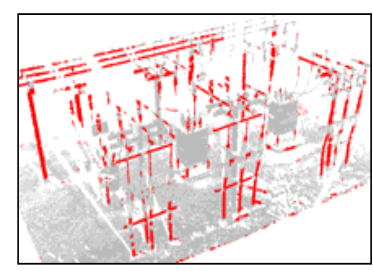

(a)

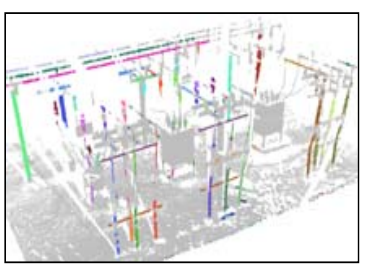

(b)
Figure 8. Electrical substation dataset: (a) detected linear/cylindrical features (red points) and (b) extracted linear/cylindrical features

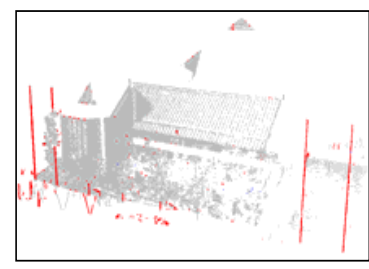

(a)

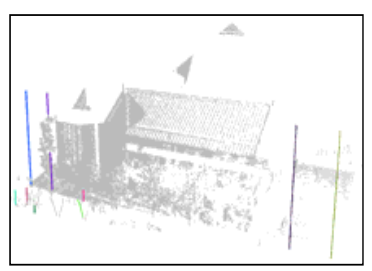

(b)
Figure 9. Complex building and surrounding area dataset: (a) detected linear/cylindrical features (red points) and (b) extracted linear/cylindrical features

In order to evaluate the outcome of the proposed linear/cylindrical feature extraction procedure, completeness and correctness quality indicators are estimated (Heipke et al., 1997) to compare the extracted results with the reference data (Equations 9 and 10).

$$
\begin{aligned}
& \text { Completeness }=\frac{T P}{T P+F N} \\
& \text { Correctness }=\frac{T P}{T P+F P}
\end{aligned}
$$

Where TP (True Positive) is a linear/cylindrical feature in the reference data which has been correctly detected using the proposed approach. A FP (False Positive) is a nonlinear/cylindrical object in the reference data which has been detected as linear/cylindrical feature. A FN (False Negative) is a linear/cylindrical object that has not been detected as linear/cylindrical feature utilizing this approach, and TN (True Negative) is a non-linear/cylindrical object in the reference data which has not been extracted using this procedure. Table 2 shows the established matrix for the quality analysis of the linear/cylindrical feature extraction results from the utilized terrestrial laser datasets (Figures 6.a and 6.b).

\begin{tabular}{|c|c|c|c|c|}
\hline & \multicolumn{2}{|c|}{$\begin{array}{c}\text { Electrical substation } \\
\text { (Reference data) }\end{array}$} & \multicolumn{2}{|c|}{$\begin{array}{r}\text { Building and surrounding } \\
\text { (Reference data) }\end{array}$} \\
\hline $\begin{array}{c}\text { Result from } \\
\text { the proposed } \\
\text { approach }\end{array}$ & Lin./cyl. & $\begin{array}{c}\text { Non- } \\
\text { lin./cyl. }\end{array}$ & Lin./cyl. & $\begin{array}{c}\text { Non- } \\
\text { lin./cyl. }\end{array}$ \\
\hline $\begin{array}{c}\text { Identified as } \\
\text { lin./cyl. features }\end{array}$ & $45(\mathrm{TP})$ & $2(\mathrm{FP})$ & $12(\mathrm{TP})$ & $4(\mathrm{FP})$ \\
\hline $\begin{array}{c}\text { Missing } \\
\text { lin./cyl. features }\end{array}$ & $10(\mathrm{FN})$ & $\mathrm{N} / \mathrm{A}(\mathrm{TN})$ & $3(\mathrm{FN})$ & N/A(TN) \\
\hline
\end{tabular}

Table 2: The quality analysis of the linear/cylindrical feature extraction process

Based on the above matrix, completeness was calculated as $81 \%$ for the electrical substation. The remaining $19 \%$ of the 
linear/cylindrical features have not been extracted due to lower point density along these objects or errors in PCA-based linear/cylindrical features detection. The correctness quality indicator was calculated as $95.7 \%$ for this dataset. $4.3 \%$ of the features, which were erroneously extracted as linear/cylindrical features, mostly belong to boundaries of planar features.

For the terrestrial laser dataset captured from the building complex and its surrounding area, completeness was calculated as $80 \% .20 \%$ of the linear/cylindrical features have not been extracted due to lower point density along these objects or cluster sizes less than the size of the smallest cluster to be identified. The correctness was calculated as $75 \%$ for this dataset. The $25 \%$ of the features, which were erroneously extracted as linear/cylindrical features, mostly belong to boundaries of planar features or edges in the dataset. The evaluation of achieved quality indicators demonstrates that the proposed methodology is able to extract the majority of the linear/cylindrical features reliably.

\section{CONCLUSIONS AND FUTURE WORK}

In this paper, a new hybrid approach for the extraction of linear/cylindrical features from laser scanning data has been introduced. The proposed procedure has two main advantages: Initial detection and modeling of the linear/cylindrical features in the spatial domain and efficient segmentation and extraction of linear/features in two low-dimensional parameter domains. In the first stage of the proposed approach, a principal component analysis of the local neighborhoods of individual laser scanning points is performed to detect and model the potential linear/cylindrical features. Afterwards, geometric attributes which describe the detected linear/cylindrical features are estimated using an iterative line/cylinder fitting procedure. The detected linear/cylindrical features are finally isolated and extracted using a parameter-domain segmentation approach. This approach overcomes the limitations of previouslydeveloped parameter-domain approaches by breaking down the attribute space into a set of subspaces and reducing the number of the geometric parameters representing linear/cylindrical features for more efficient features extraction (i.e., in lowerdimensional direction and position attribute spaces). This approach also resolves the problems of available spatial domain approaches by utilizing principal component analysis for the detection and appropriate representation, and iterative line/cylinder fitting procedure for precise modeling of the detected linear/cylindrical features.

Future research work will be focusing on the utilization of cylinder radius, which has been estimated through the iterative cylinder fitting procedure, for the discrimination of collinear linear and cylindrical features, and coaxial cylindrical features. A ground filtering procedure will also be implemented to reduce the massive amount of points to be processed for linear/cylindrical features extraction. Moreover, a quality control procedure will be developed to address different issues affecting the quality of the linear/cylindrical feature extraction from laser scanning data and propose possible actions for resolving these problems. In addition, the geometric and spectral properties of the extracted linear/cylindrical features will be incorporated in further processing steps for their interpretation and classification.

\section{ACKNOWLEDGEMENTS}

The authors would like to thank Tecterra and the Natural Sciences and Engineering Research Council of Canada (NSERC) for the financial support of this research work.

\section{REFERENCES}

Belton, D. and Lichti, D., 2006. Classification and segmentation of terrestrial laser scanner point clouds using local variance Information. The International Archives of the Photogrammetry, Remote Sensing and Spatial Information Sciences, XXXVI(5), pp. 44-49.

Bolles, R. C. and Fischler, M. A., 1981. A ransac-based approach to model fitting and its application to finding cylinders in range data. Proceedings of the 7th International Joint Conferences on Artificial Intelligence, Vancouver, Canada, pp. 637-643.

Chaperon, T. and Goulette, F., 2001. Extracting cylinders in full 3D data using a random sampling method and the gaussian image. Proceedings of Vision, Modelling and Visualisation, University of Stuttgart, Germany, pp. 35-42.

El-Halawany, S. and Lichti, D., 2011. Detection of road poles from mobile terrestrial laser scanner point cloud. Proceedings of 2011 International Workshop on Multi-Platform/MultiSensor Remote Sensing and Mapping (M2RSM), Xiamen, China, pp.1-6,

Fischler, M. A. and Bolles, R. C., 1987. Random sample consensus: A paradigm for model fitting with applications to image analysis and automated cartography. Readings in Computer Vision: Issues, Problems, Principles, and Paradigms, Los Altos, CA., USA, pp. 726-740.

Gross, H. and Thoennessen, U., 2006. Extraction of lines from laser point clouds. International Archives Of Photogrammetry, Remote Sensing And Spatial Information Sciences, XXXVI(3).

Heipke, C., Mayer, H., Wiedemann, C., and Jamet, O., 1997. Evaluation of Automatic Road Extraction. International Archives of Photogrammetry and Remote Sensing, 47-56.

Kimme, C., Ballard, D. and Sklansky, J., 1975. Finding circles by an array of accumulators. Communications of ACM, 18(2), pp. 120-122.

Luk'acs, G., Martin, R., and Marshall, D., 1998. Faithful leastsquares fitting of spheres, cylinders, cones and tori for reliable segmentation. ECCV '98: Proceedings of the 5th European Conference on Computer Vision, Springer-Verlag, I, pp. 671686.

Marshall, A. D., Luk'acs, G. and Martin, R. R., 2001. Robust segmentation of primitives from range data in the presence of geometric degeneracy. IEEE Transactions on Pattern Analysis and Machine Intelligence, 23(3), pp. 304-314.

Pauly, M., Gross, M., and Kobbelt, L. P., 2002. Efficient simplification of point-sampled surfaces. Proceedings of the Conference on Visualization, VIS '02, Washington, D.C., USA, pp. 163-170.

Rabbani, T. and van den Heuvel, F., 2005. Efficient Hough transform for automatic detection of cylinders in point clouds. Proceedings of the ISPRS Workshop Laser scanning 2005, Netherland, ISPRS Archives (XXXVI), pp.60-65.

Rabanni T., 2006. Automatic reconstruction of industrial installations using point clouds and images. PhD thesis. NCG, publication on Geodesy 62.

Vosselman, G., Gorte, B.G.H., Sithole, G., and Rabbani, T., 2004. Recognising structure in laser scanner point clouds. International Archives of the Photogrammetry, Remote Sensing and Spatial Information Sciences, 46 (part8/W2), pp. 33-38. 